\title{
EFECTO DE LA ALTURA DEL MANGUITO EN UNIONES SOLDADAS EN CAÑERÍAS DE COBRE
}

\section{BUSHING HEIGHT EFFECT IN SOLDERED COPPER PIPE JOINTS}

\author{
Víctor Carmona $^{1} \quad$ Jorge Vergara D. $^{1} \quad$ Juan Miguel Godoy R. $^{1}$ \\ Recibido 7 de marzo de 2006, aceptado el 17 de agosto de 2006 \\ Received: March 7, 2006 Accepted: August 17, 2006
}

\begin{abstract}
RESUMEN
Se perforó un tubo de cobre de $28,6 \mathrm{~mm}$ de diámetro, por el proceso de taladrado por fluencia térmica (TFT). Se prepararon manguitos de diferentes alturas, haciendo un preperforado con brocas convencionales HSS de distintos diámetros. Se seleccionaron manguitos de dos alturas diferentes. Se determinó la circularidad de la perforación. Se soldó un tubo de cobre de $\varnothing 12,7 \mathrm{~mm}$ en forma perpendicular a un tubo de cobre de ø 28,6 mm y se determinó la resistencia a la tracción de la unión soldada, para lo cual se diseñó un dispositivo mecánico que fue adaptado en la máquina universal de ensayos. Se hicieron ensayos de microdureza y metalografía de la unión. Se concluyó que el manguito de menor altura es suficiente para que la unión alcance la máxima resistencia.
\end{abstract}

Palabras clave: Manufactura de cobre, taladrado no convencional, energía no convencional, calentamiento solar.

\section{ABSTRACT}

Copper tubes were drilled with thermal flow drilling. Conventional HSS drills diameters were used to make pre drilling holes. Different height bushings were made. Two of the bushing heights were selected. The bushing circularity was measured. A $\varnothing 12,7 \mathrm{~mm}$ tube was welded perpendicularly on a ø 28,6 mm tube. A especial support device was designed and it was adapted to the Universal Test Machine, to determine the tensile stress of the brazing joint. The micro hardness and metallographic test were made in the brazing zone. As a conclusion the lower height bushing is enough to reach the maximum resistance.

Keywords: Copper manufacturing, non conventional drilling, non conventional energy, solar heating.

\section{INTRODUCCIÓN}

Uno de los procesos de unión de partes más utilizados en el campo de la mecánica es la soldadura. Hace algunos años los tubos de cobre se unían mediante soldadura plomoestaño, pero el efecto dañino del plomo ha llevado a buscar nuevos elementos para uniones soldadas. Una alternativa es la utilización de la soldadura cobre-plata.

El proceso de unión de tubos de paredes delgadas es complejo ya que existe un área de contacto muy pequeña entre los tubos a soldar. Esta zona soldada no puede ser sometida a esfuerzos de deformación o torsión por el riesgo de rotura. Este inconveniente es evitado utilizando el proceso de taladrado por fluencia térmica [1].
Este proceso consiste en realizar la perforación mediante la fricción entre la herramienta y el material a perforar. La herramienta al girar y avanzar calienta la región circundante a la perforación, haciendo fluir el material y forma un manguito o buje, la que proporciona una mayor superficie de unión y le entrega una mayor estabilidad a la unión. La herramienta utilizada para este proceso es de carburo de tungsteno, cilíndrica en su parte superior para terminar de forma cónica en la punta y provista de lóbulos [2], ver figura 1.

En el TFT, la calidad del buje, la dureza, las fuerzas de corte, el momento torsor y los mejores parámetros de trabajo han sido determinados en cobre y latón. [3]. Las modificaciones microestructuales debidas a la extrusión

1 Escuela Universitaria de Ingeniería Mecánica. Universidad de Tarapacá, 18 de Septiembre 2222 Arica, e-mail, jmgodoy@uta.cl. Arica-Chile. 
del material hacia la parte superior e inferior de una lámina durante el perforado en materiales como acero SAE 1020 y SAE 4340, aluminio A1 5052 y titanio comercialmente puro ya fueron estudiadas. [4]

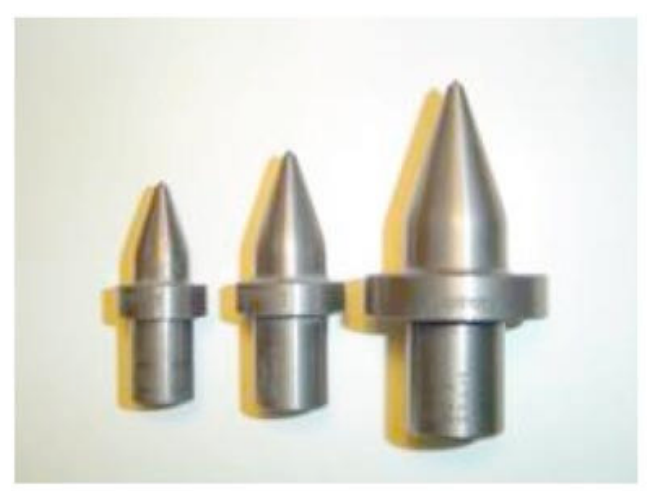

Figura 1. Brocas para tubos de $\mathrm{Cu}, 3 / 8$ ", 1/2" y 3/4".

\section{PROCEDIMIENTO EXPERIMENTAL}

\section{Perforación de los tubos de cobre}

Se perforaron tubos de cobre de 28,6 [mm] de diámetro exterior y 1,27 $\mathrm{mm}$ de espesor de la pared. Las perforaciones fueron hechas con una broca de taladrado por fluencia térmica de 12,8 [mm] de diámetro. Ensayos realizados con anterioridad demostraron que para obtener manguitos de buena calidad se debe realizar un agujero previo o preperforado con una broca convencional de acero rápido, HSS [5]. Por lo tanto, se hicieron ensayos con diámetros desde $30 \%$ hasta un $80 \%$ del diámetro equivalente de la broca de taladrado por fluencia térmica. En la tabla 1 se muestra la correspondencia entre el diámetro en milímetros de la broca y su relación porcentual con la broca de taladrado por fluencia térmica (Flowdrill).

Tabla 1. Diámetro porcentual de las brocas en comparación con la herramienta Flowdrill.

\begin{tabular}{|l|c|c|}
\hline BROCA & $\begin{array}{c}\text { DIÁMETRO } \\
(\mathbf{m m} .)\end{array}$ & RELACIÓN \% \\
\hline Flowdrill & 12,8 & $100 \%$ \\
\hline Preperforado & 4 & $30 \%$ \\
\hline Preperforado & 5 & $40 \%$ \\
\hline Preperforado & 6 & $50 \%$ \\
\hline Preperforado & 8 & $60 \%$ \\
\hline Preperforado & 9 & $70 \%$ \\
\hline Preperforado & 10 & $80 \%$ \\
\hline
\end{tabular}

Los agujeros fueron realizados con un avance $\mathrm{F}=0,1$ $[\mathrm{mm} / \mathrm{rev}]$ y con velocidades de rotación entre $\mathrm{S}=1.000$ y 4.600 [rpm].

En los agujeros preperforados se verificó una excelente calidad superficial del manguito obtenido después del taladrado por fluencia térmica.

Para preparar las uniones soldadas se utilizaron manguitos de menor y de mayor longitud obtenidos.

\section{Fabricación de los sistemas de uniones soldadas}

Los sistemas de unión se prepararon cortando tubos de cobre de 160 [mm] de largo de una cañería de 12,7 [mm] de diámetro exterior y $0,89[\mathrm{~mm}]$ de espesor de pared. Estos tubos fueron introducidos en los agujeros del tubo de 28,6 [mm] de diámetro exterior, como se observa en la figura 2.

Se emplearon cinco tipos de soldadura [6], una de cobrecobre y las otras cuatro, cobre con diferentes porcentajes de plata. La tabla 2 muestra los diferentes tipos de soldadura, se prepararon dos sistemas de unión, uno con manguito corto y otro con manguito largo.

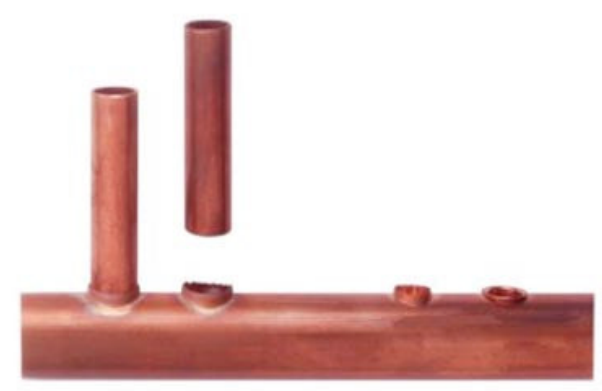

Figura 2. Proceso de soldadura de los tubos de cobre.

Tabla 2. Características de la soldadura utilizada.

\begin{tabular}{|c|c|c|c|c|c|}
\hline \multirow{2}{*}{ Aleación } & \multirow{2}{*}{$\begin{array}{l}\text { Norma } \\
\text { ANSI }\end{array}$} & \multirow{2}{*}{$\begin{array}{c}\text { Norma } \\
\text { AWS }\end{array}$} & \multicolumn{3}{|c|}{ Comp. Química } \\
\hline & & & Ag & $\mathrm{Cu}$ & $\mathbf{P}$ \\
\hline Soldargen 100 & A5.8-92 & B Cu P-2 & - & 93 & 7 \\
\hline Argenta SP2 & A5.8-92 & B Cu P-6 & 2 & 91 & 7 \\
\hline Argenta SP5 & A5.8-02 & B Cu P-7 & 5 & 88 & 7 \\
\hline Argenta SP6 & A5.8-02 & B Cu P-4 & 6 & 87 & 7 \\
\hline Argenta SP15 & A5.8-02 & B Cu P-5 & 15 & 80 & 7 \\
\hline
\end{tabular}


Las fotos de la figura 3 muestran una probeta del sistema de unión soldada para ser sometido a ensayo de tracción.

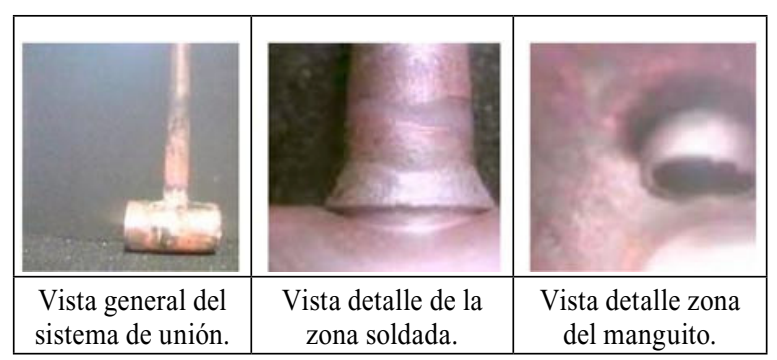

Figura 3. Detalle de las uniones soldadas.

\section{Fabricación del dispositivo mecánico}

Para confeccionar las mordazas se consideraron el sistema de sujeción de la máquina universal de ensayos y la geometría de las probetas de ensayo. También se consideró la forma de las mordazas existentes de la máquina de ensayos.

La figura 4 muestra la probeta montada en el dispositivo y éste, a su vez, en la máquina de ensayos universal.

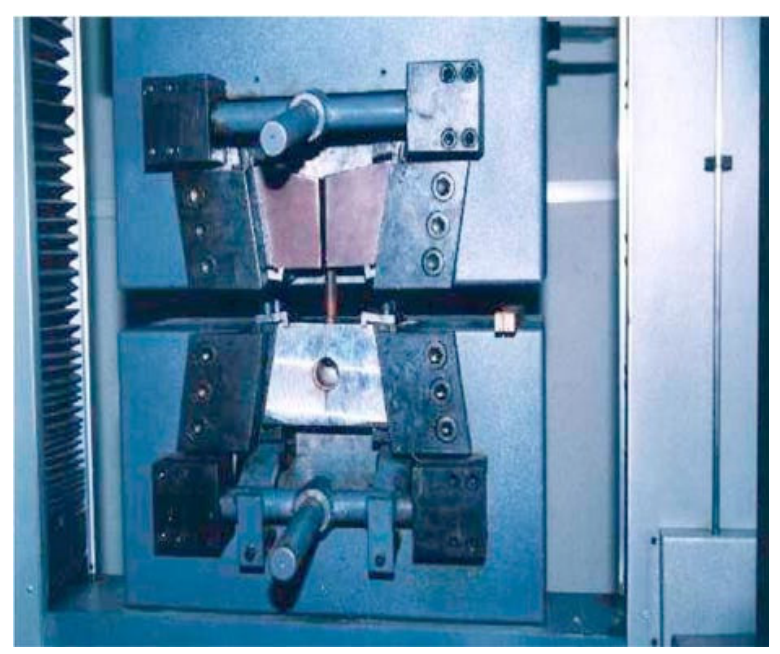

Figura 4. Detalle del dispositivo de tracción para probetas soldadas.

\section{RESULTADOS}

\section{Resistencia de la soldadura}

La fuerza máxima a la tracción de las uniones soldadas de los manguitos cortos y manguitos largos, con los diferentes tipos de soldadura, se muestran en la tabla 3.
La soldadura presenta una alta calidad en la unión, los tubos se rompieron sobre la zona soldada. Esto puede ser observado en la figura 5.

La resistencia mecánica del tubo sin soldar es de 420 MPa y después de soldado fue de 210 a $230 \mathrm{MPa}$. Valor correspondiente a un cobre recocido, que es la situación del tubo. La zona soldada trabaja en esfuerzo de corte, la resistencia teórica de la soldadura utilizada en un manguito corto corresponde a $250 \mathrm{MPa}$, valor superior al de la rotura de los tubos.

Tabla 3. Fuerzas máximas aplicadas en los ensayos de tracción.

\begin{tabular}{|c|c|c|c|c|c|}
\hline $\begin{array}{c}\text { Tipo de } \\
\text { sold. }\end{array}$ & Manguito & $\begin{array}{c}\text { Fuerza } \\
\text { máx(N) }\end{array}$ & $\begin{array}{c}\text { Tipo de } \\
\text { sold. }\end{array}$ & Manguito & $\begin{array}{c}\text { Fuerza } \\
\text { máx(N) }\end{array}$ \\
\hline SP2 & Corto & 7.430 & SP2 & Largo & 7.380 \\
\hline SP5 & Corto & 7.060 & SP5 & Largo & 7.600 \\
\hline SP6 & Corto & 7.520 & SP6 & Largo & 7.810 \\
\hline SP15 & Corto & 7.570 & SP15 & Largo & 7.350 \\
\hline $\begin{array}{c}\text { Sold. } \\
100\end{array}$ & Corto & 7.130 & $\begin{array}{c}\text { Sold. } \\
100\end{array}$ & Largo & 7.330 \\
\hline
\end{tabular}

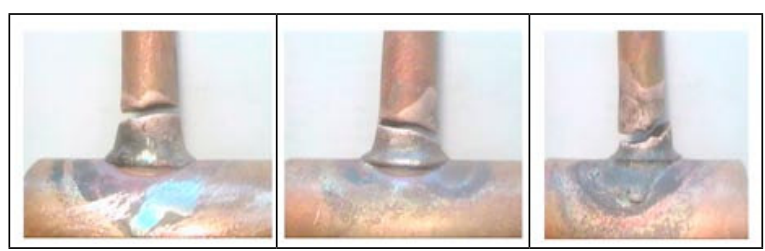

Figura 5. Zonas de rompimiento de las probetas soldadas.

\section{Macroscopia de la zona soldada}

En la figura 6 se muestra la macro y microestructura de una probeta soldada. El tamaño de grano se estima en $0,040[\mathrm{~mm}]$.

La penetración de la soldadura alcanza más de $82 \%$. La soldadura cobre-plata exige una temperatura de fusión entre $643^{\circ}$ y $802^{\circ} \mathrm{C}$ y un tiempo necesario para que todo el material se caliente.

La alta temperatura y el tiempo permitieron que toda la microestructura del cobre en torno de la soldadura recristalizara y presentase granos equiaxiales.

En la zona a se muestra la microestructura del tubo de cobre de 12,7 [mm]. Las zonas b, c, d y e, la penetración de la soldadura plata entre el tubo y manguito. La zona h muestra los cristales no deformados de la tubería de cobre de 28,6 [mm]. Las zonas $\mathbf{g}$ y $\mathbf{h}$ muestran los granos recristalizados en su forma equiaxial del manguito, la soldadura y el tubo de 12,7 [mm]. 


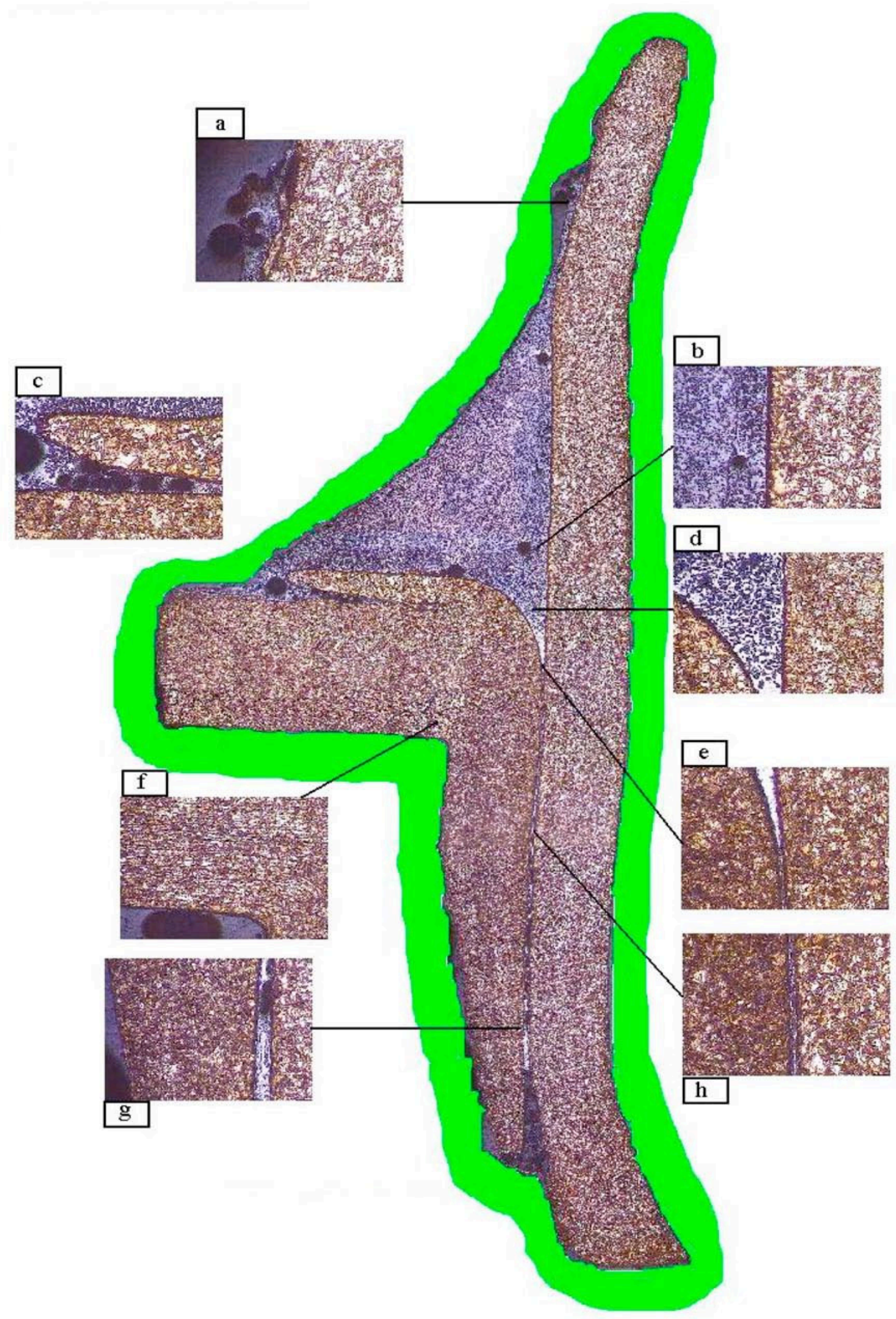

Figura 6. Micrografía de la unión soldada. 


\section{Dureza de la zona perforada}

La figura 7 es un esquema de la figura 6 y muestra las zonas que fueron sometidas al ensayo de microdureza. Estas zonas corresponden al borde externo y al centro del tubo de $12,7[\mathrm{~mm}]$.

La dureza medida en la zona soldada corresponde a un cobre recocido, producto del calentamiento durante la soldadura. La parte del tubo sin soldar indica durezas mayores, que se deben a la deformación y rompimiento del tubo durante el ensayo de tracción.

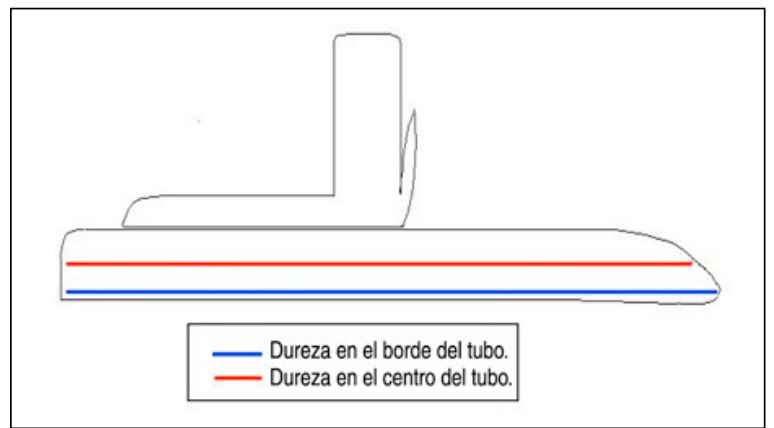

Figura 7. Diagrama de las zonas medidas con microdureza.

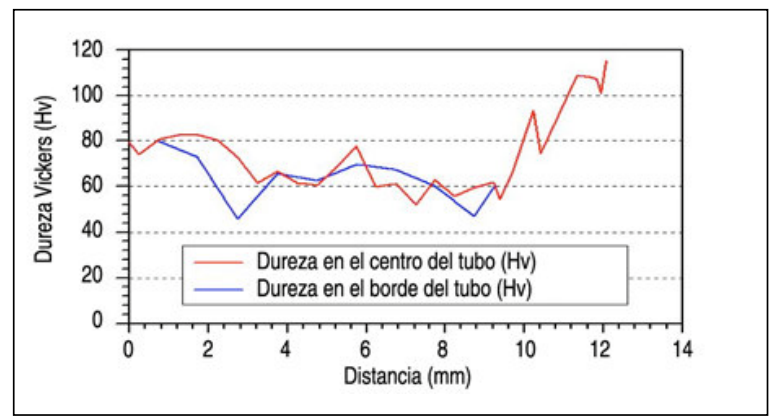

Figura 8. Dureza Vickers versus distancia.

\section{Circularidad de la perforación}

La figura 9 muestra el gráfico obtenido en el ensayo de circularidad en un tubo de cobre de 26,8 [mm]. Este gráfico indica que los agujeros realizados en el tubo de 1 " de diámetro con la herramienta para el taladrado por fluencia térmica, presentan una pequeña tendencia a la ovaladura. El punto más alejado del diámetro medio presenta una variación de un $0,32 \%$. Esto indica que la deformación del agujero no es significativa, además no presentó ninguna dificultad en el proceso de soldadura.

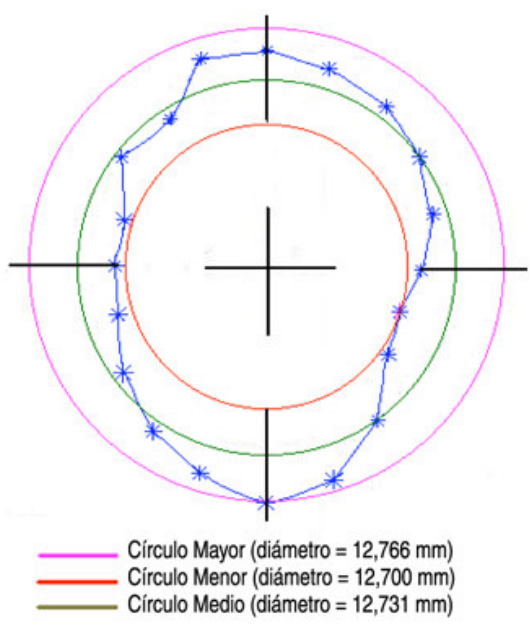

Figura 9. Gráfico de la circularidad de la perforación en tubo de cobre de 26,8 [mm] de diámetro.

\section{CONCLUSIONES}

Para obtener un manguito de buena calidad en tubos de cobre con herramienta para el taladrado por fluencia térmica, se debe realizar un preperforado, cuyo agujero hace de guía.

El ensayo de tracción demostró que para la fabricación de los sistemas de unión de cobre-cobre es suficiente un agujero con manguito corto, se puede utilizar la soldadura cobre-plata de menor precio.

El ensayo de circularidad indica que el agujero toma una forma ovalada debido a la anisotropía del material. El error porcentual de la circularidad indica que la deformación no es significativa y no afecta la unión de los tubos.

Inicialmente la estructura microscópica del tubo de cobre corresponde a granos deformados plásticamente con una dureza Vickers $\mathrm{Hv}=130$. El grano uniforme en el manguito corresponde a un cobre recocido y se debe al calor entregado durante la soldadura, lo que hace que disminuya la dureza Vickers a valores $\mathrm{Hv}=65$. Es notorio el aumento de la dureza en la proximidad de la zona de ruptura del tubo.

La resistencia a la tracción del tubo sin soldadura se reduce en un $50 \%$ después de soldado, valor correspondiente a cobre recocido. 


\section{AGRADECIMIENTOS}

Se agradece a la Universidad de Tarapacá, que financió el proyecto mayor 8741-01, responsable por este trabajo.

\section{REFERENCIAS}

[1] J.C. Oliveira. "Taladrado por fluencia y roscado por conformación”. Universidad de Santa Catarina. Florianópolis, Brasil. Abril 1994.

[2] W. Weingaertner. Revista Máquinas e Metais. $\mathrm{N}^{\mathrm{o}}$ 368, pp. 85-97. Brasil. Septiembre 1996.

[3] J. Vergara, S. Damm, J. Villanueva, J.M. Godoy and F. Tikal. "Bus Making by Thermal Flow
Drilling in Copper and Brass". The International Journal for Manufacturing Science \& Production. Vol. $4 \mathrm{~N}^{\mathrm{o}}$ 2, pp. 103-111. 2001.

[4] S. Miller, P. Blau and A. Shih. "Microestructural Alterations Associated with Friction Drilling of Steel, Aluminum and Titanium”. Journal of Materials Engineering and Performance. Vol. 14 No 5, pp. 647-653. Octubre 2005.

[5] J.C. Oliveira. "Una colaboración para la continuidad del desarrollo de la tecnología del Taladrado por Fluencia”. Tesis para optar al grado de doctor. Universidad de Kassel. Alemania. 1999.

[6] Compañía Chilena de Soldaduras Especiales, ARGENTA. 